\title{
A construction of biharmonic maps into homogeneous spaces
}

\author{
Roger Moser
}

\begin{abstract}
Biharmonic maps are the solutions of a variational problem, but they are difficult to study with variational methods, in part due to the lack of coercivity of the underlying functional. Recently Hornung was able to apply the direct method to a modified functional under the assumption that the dimension of the domain is 3 or 4. In this paper, the corresponding minimizers are studied in the case of a homogeneous target space. It is shown that they also represent minimizers of the original functional among a suitable class of comparison maps. Moreover, they solve the corresponding Euler-Lagrange equation if it is interpreted in a sufficiently weak sense.
\end{abstract}

\section{Introduction}

Biharmonic maps are the critical points of a functional involving second derivatives of maps between two Riemannian manifolds. When we want to construct them, a natural approach is to minimize the functional, say under Dirichlet boundary conditions if there is a boundary. It is not obvious, however, how to find a minimizer. Indeed, it is not obvious where to look for it, i.e., what space to work in. If we are interested in the corresponding EulerLagrange equation, then a variant of the usual Sobolev spaces seems natural. From the variational point of view, however, this space is not appropriate. It does not contain some of the obvious global minimizers, and moreover, the functional is not coercive on this space. For this reason, a variant of the Euler-Lagrange equation has been derived by Hornung and the author $[4,5]$. This equation is meaningful on a larger space and seems more suitable for the study of variational aspects of the problem. But some degree of noncoercivity of the underlying functional remains, and it is still not clear how to construct solutions.

An approach to minimizing the functional through a relaxation has been explored by Hornung [3]. He proved existence of minimizers of a relaxed 
functional. Away from a small set, these minimizers are smooth and satisfy the Euler-Lagrange equation, but it is unknown if they are global biharmonic maps in general. In the case where the target manifold is a homogeneous space, we show that they do in fact give rise to a global solution of the EulerLagrange equation in a very weak sense. That is, they satisfy a condition derived by Hornung and the author [5] that is equivalent to the EulerLagrange equation for sufficiently smooth maps. The same paper also gives some regularity results, making this the first framework suitable for both existence and regularity theory for a fairly large class of manifolds.

Suppose that $(M, g)$ is a smooth Riemannian manifold of dimension $m=3$ or 4 such that the completion $\bar{M}$ is compact with smooth boundary. Suppose that $(N,\langle\cdot, \cdot\rangle)$ is a smooth, compact Riemannian manifold without boundary. We assume that there exists a Lie group that acts transitively on $N$ by isometries (i.e., $N$ is a homogeneous space). It is convenient to assume that $N$ is isometrically embedded in a Euclidean space $\mathbb{R}^{n}$. Such an embedding always exists by the Nash embedding theorem.

We are interested in maps $u: M \rightarrow N$. Before we describe biharmonic maps, we review a few facts about a related variational problem involving the Dirichlet functional

$$
E_{1}(u)=\frac{1}{2} \int_{M}|d u|^{2} d \operatorname{vol}_{M}
$$

and giving rise to harmonic maps. Here $d u$ is the tangent map, regarded as a section of the vector bundle $T^{*} M \otimes u^{-1} T N$ over $M$, and $|\cdot|$ denotes the norm induced by $g$ and $\langle\cdot, \cdot\rangle$. We also use the notation $\langle\cdot, \cdot\rangle$ for the inner product on $T^{*} M \otimes u^{-1} T N$ and similar vector bundles. The functional is well defined on the Sobolev space

$$
W^{1,2}(M ; N)=\left\{u \in W^{1,2}\left(M ; \mathbb{R}^{n}\right): u(x) \in N \text { for almost every } x \in M\right\}
$$

There also exists an intrinsic definition (not using the embedding of $N$ in $\mathbb{R}^{n}$ ) of this space, and indeed the problem studied in this paper can be described entirely intrinsically [5]. We will, however, use the ambient space in the proof of the main result, and thus it is convenient to use this definition.

We write $\nabla$ for the covariant derivative on $T M$ and other bundles over $M$, including the pull-back bundle $u^{-1} T N$ and $T^{*} M \otimes u^{-1} T N$. The EulerLagrange equation for critical points of $E_{1}$ is then

$$
\operatorname{tr} \nabla d u=0 .
$$


Its solutions are called harmonic maps. The quantity

$$
\tau(u)=\operatorname{tr} \nabla d u
$$

is the tension field of $u$. When working with the ambient space $\mathbb{R}^{n}$, we can represent it in terms of the second fundamental form $A$ of $N$. A section $X$ of $u^{-1} T N$ can then be regarded as a section of $u^{-1} T \mathbb{R}^{n}$ as well, and the corresponding covariant derivative (which can be calculated by componentwise differentiation) is denoted by $\nabla^{0}$. Then

$$
\nabla X=\nabla^{0} X+A(X, d u)
$$

Thus if $\Delta_{0}$ denotes the component-wise Laplace-Beltrami operator (with a sign convention that makes it negative semidefinite), we have

$$
\tau(u)=\Delta_{0} u+\operatorname{tr} A(d u, d u)
$$

Biharmonic maps are the critical points of the functional

$$
E_{2}(u)=\frac{1}{2} \int_{M}|\tau(u)|^{2} d \operatorname{vol}_{M} .
$$

This functional is well-defined on

$$
W_{\text {ext }}^{2,2}(M ; N)=\left\{u \in W^{2,2}\left(M ; \mathbb{R}^{n}\right): u(x) \in N \text { for almost every } x \in M\right\}
$$

and has mostly been studied on this space. It is well defined, however, on a larger space as well, namely the space comprising all $u \in W^{1,2}(M ; N)$ with a square integrable tension field. If $\pi: E \rightarrow M$ is a vector bundle over $M$ with a bundle metric, we denote by $L^{2}(E)$ the space of all sections $X$ of $E$ with $|X| \in L^{2}(M)$. Now we define

$$
W^{\tau, 2}(M ; N)=\left\{u \in W^{1,2}(M ; N): \tau(u) \in L^{2}\left(u^{-1} T N\right)\right\} .
$$

Then $E_{2}$ is well defined on $W^{\tau, 2}(M ; N)$. In contrast to $W_{\text {ext }}^{2,2}(M ; N)$, this space is independent of the choice of the embedding of $N$ in $\mathbb{R}^{n}$, and since we regard the ambient space merely as a convenient tool, it is more desirable from a geometric point of view to work with $W^{\tau, 2}(M ; N)$. It is also of advantage from a variational point of view for reasons explained below.

The Euler-Lagrange equation for critical points of $E_{2}$ in its conventional form was derived by Jiang [6]. If $R$ denotes the Riemann curvature tensor 
on $N$, then it is

$$
\Delta \tau(u)+\operatorname{tr} R(\tau(u), d u) d u=0
$$

Here, $\Delta$ denotes the Laplace operator belonging to the covariant derivative $\nabla$ on $u^{-1} T N$. This equation has a natural weak interpretation on $W_{\text {ext }}^{2,2}(M ; N)$, although some work is necessary to write down the nonlinearities implicit in the operators $\Delta$ and $\tau[7,13]$. The equation appears meaningless, however, in $W^{\tau, 2}(M ; N)$. For this reason, a different version of the Euler-Lagrange equation has been derived by Hornung and the author $[4,5]$.

For a section $X$ of $u^{-1} T N$, define

$$
J(X)=\Delta X+\operatorname{tr} R(X, d u) d u
$$

This requires some assumptions on the regularity of $X$, but since we will eventually restrict our attention to a special case, we refer to a previous paper [5] for the details. It then turns out that for all $u \in W_{\text {ext }}^{2,2}(M ; N)$, Equation (1.2) is equivalent to the condition that

$$
\Delta\langle\tau(u), X\rangle+2 \delta\langle\tau(u), \nabla X\rangle+\langle\tau(u), J(X)\rangle=0
$$

in the distribution sense for all suitable test vector fields $X$, where $\delta$ is the $L^{2}$-adjoint of the exterior derivative $d$. In contrast to Equation (1.2), however, we can study Equation (1.4) on the space $W^{\tau, 2}(M ; N)$. This is important, because we have minimizers of $E_{2}$ that do not belong to $W_{\text {ext }}^{2,2}(M ; N)$, in particular the harmonic maps constructed by Rivière [12].

If $\Xi$ is a Killing vector field on $N$, then $X=\Xi \circ u$ is a suitable test vector field for (1.4). Moreover, it automatically satisfies $\langle\tau(u), J(X)\rangle=0$. This observation is particularly useful if the target manifold is a homogeneous space (as in this paper), because the space of Killing vector fields is then quite rich by a construction of Hélein [2].

Definition 1.1. A map $u \in W^{\tau, 2}(M ; N)$ is called very weakly biharmonic if for every Killing vector field $\Xi$ on $N$, the section $X=\Xi \circ u$ of $u^{-1} T N$ satisfies

$$
\Delta\langle\tau(u), X\rangle+2 \delta\langle\tau(u), \nabla X\rangle=0
$$

in the sense of distributional derivatives.

A regularity theory exists for a somewhat different form of this equation [4]. It is readily checked, however, that the two versions are equivalent. Due 
to supercritical nonlinearities, one cannot expect unconditional regularity results for this problem (and again a counterexample is provided by Rivière [12]). But solutions that are small in an appropriate sense are smooth. If $u \in W_{\text {ext }}^{2,2}(M ; N)$, then all the notions of weakly biharmonic maps discussed here are equivalent $[4,5]$.

While a direct minimization of the functional $E_{2}$ appears to be difficult in general, it was shown by Hornung [3] that after a modification of the functional, minimizers can be found with the direct method if $m=3$ or 4 . The functional studied by Hornung can be regarded as a relaxation of $E_{2}$. As its definition is somewhat technical, we do not give the details in this paper, but we will state some consequences of the theory in Section 2.

We will show that for a homogeneous target space, the minimizer constructed by Hornung gives rise to a very weakly biharmonic map in the sense of the above definition. The first step is to show that it is in fact a minimizer of $E_{2}$ (and not just of the relaxed functional) among a suitable class of comparison maps.

In much of the existing theory on biharmonic maps, authors have used the ambient space $\mathbb{R}^{n}$ and the nearest point projection $\pi_{N}$ onto $N$, which is defined in some tubular neighbourhood of $N$, to construct variations of a map $u: M \rightarrow N$. If $u \in W_{\text {ext }}^{2,2}(M ; N)$ and $\phi \in C_{0}^{\infty}\left(M ; \mathbb{R}^{n}\right)$, then for all $t$ in a certain neighbourhood of 0 , we have $\pi_{N} \circ(u+t \phi) \in W_{\text {ext }}^{2,2}(M ; N)$. For a critical point $u \in W_{\text {ext }}^{2,2}(M ; N)$ of $E_{2}$, we expect that

$$
\left.\frac{d}{d t}\right|_{t=0} E_{2}\left(\pi_{N} \circ(u+t \phi)\right)=0,
$$

and this condition gives rise to the Euler-Lagrange Equation (1.2). If we work in $W^{\tau, 2}(M ; N)$, then variations of this form lead to maps outside of $W^{\tau, 2}(M ; N)$ in general. Thus we need a different approach.

Let $\mathcal{F}(M ; N)$ be the set comprising all smooth maps $\Phi: M \times N \rightarrow N$ such that

- for all $x \in M$, the map $p \mapsto \Phi(x, p)$ is an isometry, and

- there exists a compact set $K \subset M$ such that $\Phi(x, p)=p$ for all $x \in$ $M \backslash K$ and all $p \in N$.

For a map $u: M \rightarrow N$ and $\Phi \in \mathcal{F}(M ; N)$, we consider $v(x)=\Phi(x, u(x))$. That is, if $\operatorname{id}_{M}$ denotes the identity map on $M$, then $v=\Phi \circ\left(\operatorname{id}_{M} \times u\right)$.

Definition 1.2. A map $u \in W^{\tau, 2}(M ; N)$ is called a weak minimizer of $E_{2}$ if $E_{2}(u) \leq E_{2}\left(\Phi \circ\left(\operatorname{id}_{M} \times u\right)\right)$ for every $\Phi \in \mathcal{F}(M ; N)$. 
We also consider smooth one-parameter families $\left(\Phi_{t}\right)_{t \in(-1,1)}$ of maps from $\mathcal{F}(M ; N)$, giving rise to variations of $u$ of the form

$$
u_{t}=\Phi_{t} \circ\left(\operatorname{id}_{M} \times u\right)
$$

Let $P_{N}: M \times N \rightarrow N$ be the projection map. Then the one-parameter family $\Phi_{t}$ also gives rise to a section $F$ of $P_{N}^{-1} T N$, defined by

$$
F(x, p)=\left.\frac{\partial}{\partial t}\right|_{t=0} \Phi_{t}(x, p),
$$

with the property that $F(x, \cdot)$ is a Killing vector field for every $x \in M$. Conversely, a smooth section of $P_{N}^{-1} T N$ with this property and with compact support generates a one-parameter family of maps in $\mathcal{F}(M ; N)$. By the construction of Hélein [2], the Killing vector fields on $N$ span every tangent space. Therefore, they can be used to construct coordinates in the tangent spaces and the above variations can be regarded as natural intrinsic counterparts to the "extrinsic" variations used in (1.6). In fact, the latter can be thought to correspond to constant vector fields in $\mathbb{R}^{n}$ in the same way. We will also see that Equation (1.5) arises from the condition $\left.\frac{d}{d t}\right|_{t=0} E_{2}\left(u_{t}\right)=0$ when $F(x, p)=\eta(x) \Xi(p)$ for a Killing vector field $\Xi$ on $N$ and a function $\eta \in C_{0}^{\infty}(M)$.

Combining all of these ingredients, we can prove the following.

Theorem 1.1. Suppose that there exists a number $c>0$ and a smooth tangent vector field $X$ on $\bar{M}$ such that at every point $x \in \bar{M}$ and for every $Y \in T_{x} \bar{M}$

$$
|Y|^{2}(\operatorname{div} X(x)-c) \geq 2 g\left(\nabla_{Y} X(x), Y\right)
$$

Then for any $u_{0} \in C^{\infty}(\bar{M} ; N)$, there exists a weak minimizer $u \in W^{\tau, 2}$ $(M ; N)$ of $E_{2}$ with $u=u_{0}$ and $d u=d u_{0}$ on $\partial M$ and such that there exists a closed, countably $(m-2)$-rectifiable set $\Sigma \subset M$ of finite $(m-2)$-dimensional Hausdorff measure with $u \in C^{\infty}(M \backslash \Sigma ; N)$.

Condition (1.8) is used to guarantee that the Dirichlet energy $E_{1}$ is controlled by $E_{2}$ and the boundary conditions with a Pohozaev type identity. It is satisfied in particular if $M$ is a subset of $\mathbb{R}^{m}$ (with $X(x)=x$ ). If the condition is not satisfied, then we still expect similar results for certain functionals that bound $E_{1}$ directly, such as $E_{2}+E_{1}$.

Since the space $W^{\tau, 2}(M ; N)$ does not provide a lot of regularity, we need to say something about the boundary conditions. Of course we can 
interpret the condition $u=u_{0}$ in the sense of traces of Sobolev spaces, since $W^{\tau, 2}(M ; N) \subset W^{1,2}(M ; N)$. Considering the extrinsic representation (1.1) of the tension field $\tau(u)$, we also see that $\Delta_{0} u \in L^{1}\left(M ; \mathbb{R}^{n}\right)$. This is enough to make sense of the normal derivative of $u$ on $\partial M$. The derivative in tangential directions is of course given by the condition $u=u_{0}$ on $\partial M$.

The proof of this result depends crucially on the work of Hornung [3]. He constructs a candidate for a biharmonic map, and we prove in this paper that it is in fact a weak minimizer of $E_{2}$. We also show that it is a very weakly biharmonic map. In fact, the following holds true.

Proposition 1.1. Suppose that $u \in W^{\tau, 2}(M ; N)$ is a weak minimizer of $E_{2}$ and there exists a closed null set $\Sigma \subset M$ such that $u \in C^{\infty}(M \backslash \Sigma)$. Then $u$ is very weakly biharmonic.

It is an open question whether all weak minimizers of $E_{2}$ in $W^{\tau, 2}(M ; N)$ are very weakly biharmonic.

In order to understand how strong the condition for a weak minimizer is, we briefly consider the space $\mathcal{F}(M ; N)$ and the transformations of a smooth map $u: M \rightarrow N$ that we can achieve by $\Phi \circ\left(\operatorname{id}_{M} \times u\right)$ with $\Phi \in \mathcal{F}(M ; N)$. It turns out that locally, we have no restrictions if $N$ is connected. More precisely, the following is true.

Proposition 1.2. Suppose that $N$ is connected. Let $u, v \in C^{\infty}(M ; N)$ and $x_{0} \in M$. Then there exists a neighbourhood $\Omega \subset M$ of $x_{0}$ and some $\Phi \in$ $\mathcal{F}(M ; N)$ such that $\left.v\right|_{\Omega}=\Phi \circ\left(\operatorname{id}_{\Omega} \times\left. u\right|_{\Omega}\right)$.

On the other hand, we do have global obstructions. An example is the following.

Proposition 1.3. Let $p_{0} \in S^{2}$ and let $u, v: S^{2} \rightarrow S^{2}$ be the maps defined by $u(x)=p_{0}$ and $v(x)=x$ for all $x \in S^{2}$. Then there exists no $\Phi \in \mathcal{F}\left(S^{2} ; S^{2}\right)$ with $v=\Phi\left(\operatorname{id}_{S^{2}} \times u\right)$.

\section{Hornung's result}

We now describe certain aspects of Hornung's work on the construction and minimization of a relaxed functional. We will not give many details, in fact we will not even give the definition of the functional, because the work depends on technically sophisticated (and perhaps somewhat exotic) tools from geometric measure theory, which would make a full description 
too lengthy for this paper. The construction can be found in Hornung's paper [3] and some of the underlying tools also in a paper by the author [8]. Both of these study flat domain manifolds, but it is not difficult to generalize the results.

If we work with the functional $E_{2}$ in the space $W^{\tau, 2}(M ; N)$, then we first observe that the condition $E_{2}(u)<\infty$ provides almost no control of the derivatives of $u$ other than $\tau(u)$. The situation improves, however, if we introduce additional conditions. These are based on the following observations. For a smooth map $u: M \rightarrow N$ and a smooth tangent vector field $X$ on $M$, define

$$
H_{X}(u)=\frac{1}{2} X\left(|d u|^{2}\right)+\delta\langle d u(X), d u\rangle+\operatorname{tr}\langle d u(\nabla X), d u\rangle .
$$

That is, if $\left(e_{1}, \ldots, e_{m}\right)$ is an orthonormal tangent frame field, then

$$
H_{X}(u)=\frac{1}{2} X\left(|d u|^{2}\right)-\sum_{\alpha=1}^{m} e_{\alpha}\left\langle d u(X), d u\left(e_{\alpha}\right)\right\rangle+\sum_{\alpha=1}^{m}\left\langle d u\left(\nabla_{e_{\alpha}} X\right), d u\left(e_{\alpha}\right)\right\rangle .
$$

Then we calculate

$$
H_{X}(u)=-\langle d u(X), \tau(u)\rangle
$$

Maps in $W^{\tau, 2}(M ; N)$ do not have this property in general, even though $H_{X}(u)$ is still well-defined as a distribution on $M$. On the other hand, if we have a map in $W^{\tau, 2}(M ; N)$ that satisfies $(2.1)$, then several useful properties can be proved. First, integrating over $M$, we obtain formally

$$
\begin{aligned}
\int_{M}\langle d u(X), \tau(u)\rangle d \operatorname{vol}_{M}= & \int_{M}\left(\frac{1}{2}|d u|^{2} \operatorname{div} X-\operatorname{tr}\langle d u(\nabla X), d u\rangle\right) d \operatorname{vol}_{M} \\
& +\int_{\partial M}\left(\langle d u(X), d u(\nu)\rangle-\frac{1}{2}|d u|^{2} g(X, \nu)\right) d \sigma_{\partial M}
\end{aligned}
$$

where $\nu$ is the outer normal vector and $\sigma_{\partial M}$ is the surface measure on $\partial M$. Under the conditions of Theorem 1.1, the boundary integral is not necessarily well defined, but using the formula, we can interpret the boundary conditions of the theorem in a stronger sense. For given boundary data, the boundary 
integral is then fixed. Moreover, under the assumption (1.8),

$$
\int_{M}\left(\frac{1}{2}|d u|^{2} \operatorname{div} X-\operatorname{tr}\langle d u(\nabla X), d u\rangle\right) d \operatorname{vol}_{M} \geq c E_{1}(u) .
$$

Using the Cauchy-Schwarz inequality, we then obtain an estimate for $E_{1}(u)$ in terms of $E_{2}(u)$ and the boundary values. Second, we can derive a generalization of a well-known monotonicity formula for harmonic maps, which has been proved (for harmonic maps) by Price [11] and Große-Brauckmann [1]. For $m \leq 4$ and for a flat domain, a similar formula has been derived by the author when $\tau(u) \neq 0[9$, Lemma 4.1], and a combination of these methods will yield a version for the situation studied here. Based on this, we then obtain some control of $d u$ for maps with finite energy similar to the usual Sobolev inequality [10]. This suggests that we should look for minimizers of $E_{2}$ in a space of maps which satisfy (2.1). Unfortunately, the equation is not preserved under weak limits. Thus if we apply the direct method in the calculus of variations, there is no guarantee that we will stay in a space defined in terms of (2.1).

The idea to overcome this difficulty is to supplement each map with a Radon measure that compensates for the failure to satisfy (2.1). The nature of the problem is such that these Radon measures can be thought of as generalized $(m-2)$-dimensional submanifolds of $M$. In particular, they are supported on countably $(m-2)$-rectifiable subsets. They also have a generalized mean curvature vector, giving rise to what may be thought of as their Willmore energy. Adding this to $E_{2}$, we obtain a functional that we can now try to minimize.

Using a further analysis of the structure of these measures, Hornung was able to achieve minimization with the direct method. The following is a direct consequence of his results [3, Theorem 2], at least for a domain in $\mathbb{R}^{m}$. For other manifolds, the statement can be proved with exactly the same methods.

Theorem 2.1. Suppose that $M$ satisfies the hypothesis of Theorem 1.1. Let $u_{0} \in C^{\infty}(\bar{M} ; N)$. Then there exists a map $u \in W^{\tau, 2}(M ; N)$ and a closed and countably $(m-2)$-rectifiable set $\Sigma \subset M$ of finite $(m-2)$-dimensional Hausdorff measure such that

(1) $u=u_{0}$ and $d u=d u_{0}$ on $\partial \Omega$,

(2) $u \in C^{\infty}(M \backslash \Sigma ; N)$,

(3) $u$ is biharmonic in $M \backslash \Sigma$, and 
(4) if $v \in W^{\tau, 2}(M ; N)$ is such that

- there exists a compact set $K \subset M$ such that $v=u$ in $M \backslash K$ and

- for all smooth tangent vector fields $X$ on $M$ with compact support,

$$
H_{X}(v)+\langle d v(X), \tau(v)\rangle=H_{X}(u)+\langle d u(X), \tau(u)\rangle,
$$

then $E_{2}(u) \leq E_{2}(v)$.

So even if $u$ is constructed as a minimizer of a different functional, it still minimizes $E_{2}$ among all maps satisfying the conditions of statement 2.1. But the conditions in this theorem are rather unwieldy, which is why we want to prove Theorem 1.1. In particular, we want to show that variations of the type (1.7) give rise to comparison maps as in the last statement of Theorem 2.1.

Hornung also proved other properties of his minimizers. He considered boundary conditions which are stronger than what is stated here, taking also his version of (2.1) into account. Furthermore, he showed that if $\Sigma$ and the corresponding Radon measure are sufficiently regular, then $u$ is a minimizer of $E_{2}$ among all maps in $W^{\tau, 2}(M ; N)$ satisfying $(2.1)$ and coinciding with $u$ outside of a compact set. The tools developed for this purpose are also useful in the context of Theorem 1.1. In particular, if we have sufficient regularity of $\Sigma$ and the corresponding Radon measure, then it follows that $u$ is smooth away from a closed singular set of vanishing $(m-2)$-dimensional Hausdorff measure by the regularity theory for very weakly biharmonic maps into homogeneous spaces [4].

\section{Some computations}

The purpose of this section is to prove the following statement.

Lemma 3.1. Suppose that $u \in W^{\tau, 2}(M ; N)$ such that there exists a closed null set $\Sigma \subset M$ with $u \in C^{\infty}(M \backslash \Sigma ; N)$. Let $\Phi \in \mathcal{F}(M ; N)$ and $v=\Phi \circ$ $\left(\operatorname{id}_{M} \times u\right)$. Then $v \in W^{\tau, 2}(M ; N)$, and for every smooth tangent vector field $X$ on $M$ with compact support,

$$
H_{X}(v)+\langle d v(X), \tau(v)\rangle=H_{X}(u)+\langle d u(X), \tau(u)\rangle .
$$

Proof. We first introduce some notation. Let $(x, p) \in M \times N$ be a generic point. Then we write $d \Phi(x, p)$ for the tangent map of $\Phi(\cdot, p)$ at the point $x \in M$ and $T \Phi(x, p)$ for the tangent map of $\Phi(x, \cdot)$ at the point $p \in N$. Suppose that $D^{M \times N}$ denotes the covariant derivative on a vector bundle 
$\pi: E \rightarrow M \times N$ over $M \times N$ (such as $\left.\Phi^{-1} T N\right)$. Then for tangent vectors $X$ and $Y$ on $M$ and $N$, respectively, and for a section $Z$ of $E$, we write $\nabla_{X} Z=D_{(X, 0)}^{M \times N} Z$ and $D_{Y} Z=D_{(0, Y)}^{M \times N} Z$. The Hessian of $\Phi$ then comprises $\nabla d \Phi, D T \Phi$, and the mixed derivatives, which are denoted interchangeably by $\nabla T \Phi$ and $D d \Phi$.

Recall that $N$ is embedded in $\mathbb{R}^{n}$. We extend $\Phi$ smoothly to $M \times \mathbb{R}^{n}$ such that in a neighbourhood of $N$, we have $\Phi(x, p)=\Phi\left(x, \pi_{N}(p)\right)$. We then use similar notation for the derivatives of the extension, but with $\nabla^{0}$ and $D^{0}$ for the covariant derivatives on $M$ and $N$, respectively. We use the notation $\Delta \Phi=\operatorname{tr} \nabla d \Phi$ and $\Delta_{0} \Phi=\operatorname{tr} \nabla^{0} d \Phi$.

We first consider a smooth map $\tilde{u}: M \rightarrow \mathbb{R}^{n}$ and $\tilde{v}(x)=\Phi(x, \tilde{u}(x))$. We define $\tilde{U}=\operatorname{id}_{M} \times \tilde{u}$, so that we can also write $\tilde{v}=\Phi \circ \tilde{U}$. We calculate

$$
d \tilde{v}=d \Phi(\tilde{U})+T \Phi(\tilde{U}) d \tilde{u}
$$

and

$(3.1)$

$\Delta_{0} \tilde{v}=\Delta_{0} \Phi(\tilde{U})+2 \operatorname{tr} D^{0} d \Phi(\tilde{U})(\cdot, d \tilde{u})+\operatorname{tr} D^{0} T \Phi(\tilde{U})(d \tilde{u}, d \tilde{u})+T \Phi(\tilde{U}) \Delta_{0} \tilde{u}$.

If we have a map $u \in W^{\tau, 2}(M ; N)$, then in particular $u \in W^{1,2}\left(M ; \mathbb{R}^{n}\right)$ and $\Delta_{0} u \in L^{1}\left(M ; \mathbb{R}^{n}\right)$. Using convolution with a Dirac sequence, we can therefore construct a sequence $\left(u_{k}\right)_{k \in \mathbb{N}}$ of smooth maps $u_{k}: M \rightarrow \mathbb{R}^{n}$ such that $u_{k} \rightarrow u$ strongly in $W^{1,2}\left(M ; \mathbb{R}^{n}\right)$ and $\Delta_{0} u_{k} \rightarrow \Delta_{0} u$ strongly in $L^{1}\left(M ; \mathbb{R}^{n}\right)$ as $k \rightarrow \infty$. Moreover, this sequence will be bounded in $L^{\infty}\left(M ; \mathbb{R}^{n}\right)$ and can be chosen such that we have point-wise convergence almost everywhere. Substituting $u_{k}$ for $\tilde{u}$ in (3.1) and applying Lebesgue's convergence theorem, we obtain

$$
\Delta_{0} v=\Delta_{0} \Phi(U)+2 \operatorname{tr} D^{0} d \Phi(U)(\cdot, d u)+\operatorname{tr} D^{0} T \Phi(U)(d u, d u)+T \Phi(U) \Delta_{0} u,
$$

where $U=\operatorname{id}_{M} \times u$.

Because we have extended $\Phi$ in a specific way, we have $T \Phi(x, p) \nu=0$ for any vector $\nu \in T_{p} \mathbb{R}^{n}$ normal to $N$ at $p$. Moreover, since $\left.\Phi\right|_{N}$ is an isometry, we conclude that $D^{0} T \Phi$ maps any pair of tangent vectors at $p \in N$ to a normal vector at $\Phi(x, p)$. Projecting orthogonally onto the tangent spaces, we obtain

$$
\tau(v)=\tau(\Phi)(U)+2 \operatorname{tr} D d \Phi(U)(\cdot, d u)+T \Phi(U) \tau(u)
$$

where $\tau(\Phi)(x, p)$ is the tension field of the map $\Phi(\cdot, p)$ at the point $x \in M$. The right-hand side clearly belongs to $L^{2}\left(v^{-1} T N\right)$. Thus $v \in W^{\tau, 2}(M ; N)$. 
Let $X, Y$ be smooth tangent vector fields on $M$ with compact support. Studying the smooth maps $\tilde{u}: M \rightarrow \mathbb{R}^{n}$ and $\tilde{v}=\Phi \circ \tilde{U}$ again, we calculate

$$
\begin{aligned}
\langle d \tilde{v}(X), d \tilde{v}(Y)\rangle= & \langle d \Phi(\tilde{U}) X, d \Phi(\tilde{U}) Y\rangle+\langle d \Phi(\tilde{U}) X, T \Phi(\tilde{U}) d \tilde{u}(Y)\rangle \\
& +\langle d \Phi(\tilde{U}) Y, T \Phi(\tilde{U}) d \tilde{u}(X)\rangle \\
& +\langle T \Phi(\tilde{U}) d \tilde{u}(X), T \Phi(\tilde{U}) d \tilde{u}(Y)\rangle
\end{aligned}
$$

and

$$
\begin{aligned}
\left\langle d \tilde{v}(X), \Delta_{0} \tilde{v}\right\rangle= & \left\langle d \Phi(\tilde{U}) X, \Delta_{0} \Phi(\tilde{U})\right\rangle+2\left\langle d \Phi(\tilde{U}) X, \operatorname{tr} D^{0} d \Phi(\tilde{U})(\cdot, d \tilde{u})\right\rangle \\
& +\left\langle d \Phi(\tilde{U}) X, \operatorname{tr} D^{0} T \Phi(\tilde{U})(d \tilde{u}, d \tilde{u})\right\rangle \\
& +\left\langle d \Phi(\tilde{U}) X, T \Phi(\tilde{U}) \Delta_{0} \tilde{u}\right\rangle+\left\langle T \Phi(\tilde{U}) d \tilde{u}(X), \Delta_{0} \Phi(\tilde{U})\right\rangle \\
& +2\left\langle T \Phi(\tilde{U}) d \tilde{u}(X), \operatorname{tr} D^{0} d \Phi(\tilde{U})(\cdot, d \tilde{u})\right\rangle \\
& +\left\langle T \Phi(\tilde{U}) d \tilde{u}(X), \operatorname{tr} D^{0} T \Phi(\tilde{U})(d \tilde{u}, d \tilde{u})\right\rangle \\
& +\left\langle T \Phi(\tilde{U}) d \tilde{u}(X), T \Phi(\tilde{U}) \Delta_{0} \tilde{u}\right\rangle
\end{aligned}
$$

Differentiating the terms in (3.3) and taking advantage of some cancellation, we compute

$$
\begin{aligned}
H_{X}(\tilde{v})+\left\langle d \tilde{v}(X), \Delta_{0} \tilde{v}\right\rangle=\operatorname{tr} & \left\langle D^{0} d \Phi(\tilde{U})(X, d \tilde{u}), T \Phi(\tilde{U}) d \tilde{u}\right\rangle \\
& +\operatorname{tr}\left\langle D^{0} d \Phi(\tilde{U})(\cdot, d \tilde{u}(X)), T \Phi(\tilde{U}) d \tilde{u}\right\rangle \\
& +\left\langle T \Phi(\tilde{U}) d \tilde{u}(X), \operatorname{tr} D^{0} d \Phi(\tilde{U})(\cdot, d \tilde{u})\right\rangle \\
& +\left\langle T \Phi(\tilde{U}) d \tilde{u}(X), \operatorname{tr} D^{0} T \Phi(\tilde{U})(d \tilde{u}, d \tilde{u})\right\rangle \\
& +\frac{1}{2} X\left(|T \Phi(\tilde{U}) d \tilde{u}|^{2}\right) \\
& +\delta\langle T \Phi(\tilde{U}) d \tilde{u}(X), T \Phi(\tilde{U}) d \tilde{u}\rangle \\
& +\operatorname{tr}\langle T \Phi(\tilde{U}) d \tilde{u}(\nabla X), T \Phi(\tilde{U}) d \tilde{u}\rangle \\
& +\left\langle T \Phi(\tilde{U}) d \tilde{u}(X), T \Phi(\tilde{U}) \Delta_{0} \tilde{u}\right\rangle .
\end{aligned}
$$


Now suppose that $u$ satisfies the hypotheses of the lemma and consider the approximation by smooth maps $u_{k}: M \rightarrow \mathbb{R}^{n}$ again with the properties stated above. Then (3.5) holds with $u_{k}$ instead of $\tilde{u}$. We immediately see that the terms

$$
\begin{aligned}
\operatorname{tr} & \left\langle D^{0} d \Phi\left(U_{k}\right)\left(X, d u_{k}\right), T \Phi\left(U_{k}\right) d u_{k}\right\rangle \\
& +\operatorname{tr}\left\langle D^{0} d \Phi\left(U_{k}\right)\left(\cdot, d u_{k}(X)\right), T \Phi\left(U_{k}\right) d u_{k}\right\rangle \\
& +\left\langle T \Phi\left(U_{k}\right) d u_{k}(X), \operatorname{tr} D^{0} d \Phi\left(U_{k}\right)\left(\cdot, d u_{k}\right)\right\rangle
\end{aligned}
$$

(for $U_{k}=\mathrm{id}_{M} \times u_{k}$ ) converge in $L^{1}(M)$ to the corresponding expressions involving $u$. Similarly, we have

$$
\operatorname{tr}\left\langle T \Phi\left(U_{k}\right) d u_{k}(\nabla X), T \Phi\left(U_{k}\right) d u_{k}\right\rangle \rightarrow \operatorname{tr}\langle T \Phi(U) d u(\nabla X), T \Phi(U) d u\rangle
$$

in $L^{1}(M)$. Using (3.4) for $u_{k}$ instead of $\tilde{u}$, we also see that

$$
\begin{aligned}
& \left\langle T \Phi\left(U_{k}\right) d u_{k}(X), \operatorname{tr} D^{0} T \Phi\left(U_{k}\right)\left(d u_{k}, d u_{k}\right)\right\rangle+\left\langle T \Phi\left(U_{k}\right) d u_{k}(X), T \Phi\left(U_{k}\right) \Delta_{0} u_{k}\right\rangle \\
& \quad-\left\langle d\left(\Phi \circ U_{k}\right)(X), \Delta_{0}\left(\Phi \circ U_{k}\right)\right\rangle
\end{aligned}
$$

is convergent in $L^{1}(M)$. Let $a$ be its limit. The other terms in (3.5) are distributional derivatives of expressions that converge in $L^{1}(M)$ as well, and so we have convergence as distributions. Namely, we have

$$
\begin{aligned}
H_{X}\left(v_{k}\right) & \rightarrow H_{X}(v), \\
\frac{1}{2} X\left(\left|T \Phi\left(U_{k}\right) d u_{k}\right|^{2}\right) & \rightarrow \frac{1}{2} X\left(|T \Phi(U) d u|^{2}\right), \\
\delta\left\langle T \Phi\left(U_{k}\right) d u_{k}(X), T \Phi\left(U_{k}\right) d u_{k}\right\rangle & \rightarrow \delta\langle T \Phi(U) d u(X), T \Phi(U) d u\rangle .
\end{aligned}
$$

Hence, we obtain the formula

$$
\begin{aligned}
H_{X}(v)= & \operatorname{tr}\langle D d \Phi(U)(X, d u), T \Phi(U) d u\rangle \\
& +\operatorname{tr}\langle D d \Phi(U)(\cdot, d u(X)), T \Phi(U) d u\rangle \\
& +\langle T \Phi(U) d u(X), \operatorname{tr} D d \Phi(U)(\cdot, d u)\rangle \\
& +\frac{1}{2} X\left(|T \Phi(U) d u|^{2}\right)+\delta\langle T \Phi(U) d u(X), T \Phi(U) d u\rangle \\
& +\operatorname{tr}\langle T \Phi(U) d u(\nabla X), T \Phi(U) d u\rangle+a .
\end{aligned}
$$

Since $\Phi(x, \cdot)$ is an isometry for every $x \in M$ (if restricted to $N$ ), we have

$$
\langle Y, Z\rangle=\langle T \Phi(x, p) Y, T \Phi(x, p) Z\rangle
$$


for all $p \in N$ and $Y, Z \in T_{p} N$. Let $X \in T_{x} M$ and extend $Y$ and $Z$ to sections of $\Phi^{-1} T N$ such that $\nabla_{X} Y=\nabla_{X} Z=0$ at $(x, p)$. Then

$$
\begin{aligned}
0 & =X\langle T \Phi(x, p) Y, T \Phi(x, p) Z\rangle \\
& =\langle\nabla T \Phi(x, p)(X, Y), T \Phi(x, p) Z\rangle+\langle T \Phi(x, p) Y, \nabla T \Phi(x, p)(X, Z)\rangle \\
& =\langle D d \Phi(x, p)(X, Y), T \Phi(x, p) Z\rangle+\langle T \Phi(x, p) Y, D d \Phi(x, p)(X, Z)\rangle .
\end{aligned}
$$

It follows that

$$
\begin{aligned}
& \operatorname{tr}\langle D d \Phi(U)(X, d u), T \Phi(U) d u\rangle+\operatorname{tr}\langle D d \Phi(U)(\cdot, d u(X)), T \Phi(U) d u\rangle \\
& \quad+\langle T \Phi(U) d u(X), \operatorname{tr} D d \Phi(U)(\cdot, d u)\rangle=0 .
\end{aligned}
$$

Furthermore,

$$
\begin{aligned}
\langle T \Phi(U) d u(X), T \Phi(U) d u\rangle & =\langle d u(X), d u\rangle, \\
|T \Phi(U) d u|^{2} & =|d u|^{2}, \\
\langle T \Phi(U) d u(\nabla X), T \Phi(U) d u\rangle & =\langle d u(\nabla X), d u\rangle .
\end{aligned}
$$

We infer that

$$
H_{X}(v)-H_{X}(u)=a .
$$

Recall that the right-hand side is a function in $L^{1}(M)$. The left-hand side can be calculated in $M \backslash \Sigma$, where $u$ is smooth, and coincides with

$$
\langle d u(X), \tau(u)\rangle-\langle d v(X), \tau(v)\rangle .
$$

Hence,

$$
a=\langle d u(X), \tau(u)\rangle-\langle d v(X), \tau(v)\rangle
$$

in $M$, which proves the second claim of the lemma.

\section{Proofs}

We now have all the tools for the proofs of the main results. In particular, for the proof of Theorem 1.1, it suffices to combine the previous statements. Proof of Theorem 1.1. Let $u_{0} \in C^{\infty}(\bar{M} ; N)$ and let $u \in W^{\tau, 2}(M ; N)$ be the corresponding map from Theorem 2.1. Then there exists a closed null set $\Sigma \subset M$ such that $u$ is smooth away from $\Sigma$. Combining Lemma 3.1 with Theorem 2.1, we conclude that $u$ is a weak minimizer of $E_{2}$. The boundary conditions and the partial regularity are of course the same as in Theorem 2.1. 
Proof of Proposition 1.1. Suppose that $u \in W^{\tau, 2}(M ; N)$ is a weak minimizer of $E_{2}$. Consider a smooth family of maps $\Phi_{t} \in \mathcal{F}(M ; N)$ for $-1<t<1$ such that there exists a compact set $K \subset M$ with $\Phi_{t}(x, p)=p$ for all $x \in M \backslash K$ and all $t \in(-1,1)$. Let

$$
F(x, p)=\left.\frac{\partial}{\partial t}\right|_{t=0} \Phi_{t}(x, p)
$$

and $Y(x)=F(x, u(x))$. Define $u_{t}(x)=\Phi_{t}(x, u(x))$. We first study this variation of $u$ away from $\Sigma$ (where $u$ is smooth). Then as in (3.2), we compute

$$
\tau\left(u_{t}\right)=\tau\left(\Phi_{t}\right)(U)+2 \operatorname{tr} D d \Phi_{t}(U)(\cdot, d u)+T \Phi_{t}(U) \tau(u)
$$

where once again, we write $U=\operatorname{id}_{M} \times u$. Thus there exists a constant $C_{1}$, independent of $u$, such that for all $t \in\left[-\frac{1}{2}, \frac{1}{2}\right]$,

$$
\left|\tau\left(u_{t}\right)\right| \leq C_{1}(|\tau(u)|+|d u|+1)
$$

Moreover, a direct calculation shows that

$$
\Delta Y=\operatorname{tr} \nabla^{2} F(U)+2 \operatorname{tr} D \nabla F(U)(\cdot, d u)+D_{\tau(u)} F(U)+\operatorname{tr} D^{2} F(U)(d u, d u)
$$

Since for $(x, p) \in M \times N$ and $Y, Z \in T_{p} N$, we have

$$
\begin{aligned}
D^{2} F(x, p)(Y, Z) & =\left.D^{2} \frac{\partial}{\partial t}\right|_{t=0} \Phi_{t}(x, p)(Y, Z)=\left.D \nabla_{t}\right|_{t=0} T \Phi_{t}(x, p)(Y, Z) \\
& =\left.\nabla_{t}\right|_{t=0} D T \Phi_{t}(x, p)(Y, Z)-R(F(x, p), Y) Z
\end{aligned}
$$

and $D T \Phi_{t}=0$, we conclude that

$$
J(Y)=\operatorname{tr} \nabla^{2} F(U)+2 \operatorname{tr} D \nabla F(U)(\cdot, d u)+D_{\tau(u)} F(U) .
$$

(Recall (1.3) for the definition of $J(Y)$.) Hence, there exists a constant $C_{2}$, independent of $u$, such that

$$
|J(Y)| \leq C_{2}(|\tau(u)|+|d u|+1) .
$$

Still working in $M \backslash \Sigma$, we find

$$
\left.\frac{1}{2} \frac{\partial}{\partial t}\right|_{t=0}\left|\tau\left(u_{t}\right)\right|^{2}=\langle\tau(u), J(Y)\rangle
$$


with standard computations as used by Jiang [6]. Thus

$$
\left|\frac{1}{2} \frac{\partial}{\partial t}\right|_{t=0}\left|\tau\left(u_{t}\right)\right|^{2} \mid \leq C_{2}\left(2|\tau(u)|^{2}+\frac{1}{2}|d u|^{2}+\frac{1}{2}\right) .
$$

Applying the above calculations to $u_{t}$ instead of $u$, we obtain a constant $C_{3}$ such that uniformly in $t$, we have

$$
\left.\left|\frac{1}{2} \frac{\partial}{\partial t}\right| \tau\left(u_{t}\right)\right|^{2} \mid \leq C_{3}\left(|\tau(u)|^{2}+|d u|^{2}+1\right) .
$$

The above inequality holds in $M \backslash \Sigma$, which is almost everywhere. Now it follows from Lebesgue's convergence theorem that

$$
\frac{1}{2} \lim _{t \rightarrow 0}\left(\frac{1}{t} \int_{M}\left(\left|\tau\left(u_{t}\right)\right|^{2}-|\tau(u)|^{2}\right) d \operatorname{vol}_{M}\right)=\int_{M}\langle\tau(u), J(Y)\rangle d \operatorname{vol}_{M}
$$

Since $u$ is a weak minimizer of $E_{2}$, this quantity must vanish.

If we have a Killing vector field $\Xi$ on $N$ and a function $\eta \in C_{0}^{\infty}(M)$, then we consider $F(x, p)=\eta(x) \Xi(p)$ and $Y(x)=F(x, u(x))$. Then we have

$$
J(Y)=\Delta \eta \Xi(u)+2 g\left(d \eta, D_{d u} \Xi(u)\right)+\eta D_{\tau(u)} \Xi(u) .
$$

Since $\Xi$ is a Killing vector field, we have $\left\langle\tau(u), D_{\tau(u)} \Xi(u)\right\rangle=0$. It follows that for $X=\Xi \circ u$, we have

$$
\begin{aligned}
0 & =\int_{M}\langle\tau(u), J(Y)\rangle d \operatorname{vol}_{M} \\
& =\int_{M}\left(\Delta \eta\langle\tau(u), \Xi(u)\rangle+2 g\left(d \eta,\left\langle\tau(u), D_{d u} \Xi(u)\right\rangle\right)\right) d \operatorname{vol}_{M} \\
& =\int_{M}(\Delta \eta\langle\tau(u), X\rangle+2 g(d \eta,\langle\tau(u), \nabla X\rangle)) d \operatorname{vol}_{M} .
\end{aligned}
$$

Hence, $u$ is a very weakly biharmonic map.

Proof of Proposition 1.2. Set $p_{0}=u\left(x_{0}\right)$. We first show that the statement is true if $v\left(x_{0}\right)$ is sufficiently close to $p_{0}$.

Let $k$ be the dimension of $N$. By a result of Hélein [2, Lemma 2], there exist Killing vector fields $\Xi_{1}, \ldots, \Xi_{k}$ on $N$ which give rise to a basis of every tangent space $T_{p} N$ for $p$ in a certain open neighbourhood $W \subset N$ of $p_{0}$. Choose two open sets $\Omega, \Omega^{\prime} \subset M$ with $x_{0} \in \Omega$ and $\bar{\Omega} \subset \Omega^{\prime}$. Choose a cutoff function $\eta \in C_{0}^{\infty}\left(\Omega^{\prime}\right)$ with $\eta \equiv 1$ in $\Omega$ and set $X_{i}(x, p)=\eta(x) \Xi_{i}(p)$. For 
$\alpha \in \mathbb{R}^{k}$, let $\Phi_{t}(\cdot ; \alpha)$ be the one-parameter family in $\mathcal{F}(M ; N)$ generated by $\sum_{i=1}^{k} \alpha_{i} X_{i}$, i.e., the solution of

$$
\dot{\Phi}_{t}(x, p ; \alpha)=\sum_{i=1}^{k} \alpha_{i} X_{i}\left(x, \Phi_{t}(x, p ; \alpha)\right), \quad \Phi_{0}(x, p ; \alpha)=p .
$$

Then

$$
\frac{\partial}{\partial \alpha_{i}} \Phi_{1}(x, p ; 0)=X_{i}(x, p)
$$

Moreover, we have $\Phi_{1}(x, p ; 0)=p$. By the implicit function theorem, there exists a smooth map $A: \Omega \times W \times W \rightarrow \mathbb{R}^{k}$ such that

$$
\Phi_{1}(x, p ; A(x, p, q))=q
$$

for all $x \in \Omega$ and $p, q \in W$, provided that $\Omega$ and $W$ are sufficiently small. Hence for $\Phi(x, p)=\Phi_{1}(x, p ; A(x, u(x), v(x)))$, we have the required identity as long as $u(x), v(x) \in W$ for all $x \in \Omega$.

We now define a relation $\sim$ on $N$, defined as follows. For $p, q \in N$, we write $p \sim q$ if for all smooth functions $u, v: M \rightarrow N$ with $u\left(x_{0}\right)=p$ and $v\left(x_{0}\right)=q$, there exists a neighbourhood $\Omega \subset M$ of $x_{0}$ and a $\Phi \in \mathcal{F}(M ; N)$ such that $\left.v\right|_{\Omega}=\Phi \circ\left(\operatorname{id}_{\Omega} \times\left. u\right|_{\Omega}\right)$. Then by the previous argument, the relation is reflexive. It is clearly transitive and symmetric, thus we have an equivalence relation. The above arguments also imply that the equivalence classes are open. Hence if $N$ is connected, there exists exactly one equivalence class.

Proof of Proposition 1.3. We argue by contradiction. Suppose we had a $\Phi \in$ $\mathcal{F}\left(S^{2} ; S^{2}\right)$ with $v=\Phi \circ\left(\operatorname{id}_{S^{2}} \times u\right)$. Fix a tangent vector $X_{0} \in T_{p_{0}} S^{2}$ and for every $x \in S^{2}$, define

$$
X(x)=T \Phi\left(x, p_{0}\right) X_{0}
$$

Since $\Phi(x, \cdot)$ is an isometry with $\Phi\left(x, p_{0}\right)=x$, we have $X(x) \in T_{x} S^{2}$ and $X(x) \neq 0$ for every point $x \in S^{2}$. It is well-known that no such tangent vector field exists on $S^{2}$.

Acknowledgment. This work was partially supported by the EPSRC through grant EP/F048769/1.

\section{References}

[1] K. Große-Brauckmann, Interior and boundary monotonicity formulas for stationary harmonic maps, Manuscripta Math. 77 (1992), 89-95. 
[2] F. Hélein, Regularity of weakly harmonic maps from a surface into a manifold with symmetries, Manuscripta Math. 70 (1991), 203-218.

[3] P. Hornung, A relaxation of the intrinsic biharmonic energy, Math. Z. 271 (2012), 663-692.

[4] P. Hornung and R. Moser, Intrinsically biharmonic maps into homogeneous spaces, Adv. Calc. Var. 5 (2012), 411-425.

[5] _ A reformulation of the biharmonic map equation, J. Geom. Anal. (Online First) (2012).

[6] G.Y. Jiang, 2-harmonic maps and their first and second variational formulas, Chinese Ann. Math. Ser. A 7 (1986), 389-402.

[7] R. Moser, The blow-up behavior of the biharmonic map heat flow in four dimensions, IMRP Int. Math. Res. Pap. 2005 (2005), 351-402.

[8] Energy concentration for almost harmonic maps and the Willmore functional, Math. Z. 251 (2005), 293-311.

[9] _ Partial regularity for harmonic maps and related problems, World Scientific Publishing, Singapore, 2005.

[10] _ Remarks on the regularity of biharmonic maps in four dimensions, Commun. Pure Appl. Math. 59 (2006), 317-329.

[11] P. Price, A monotonicity formula for Yang-Mills fields, Manuscripta Math. 43 (1983), 131-166.

[12] T. Rivière, Everywhere discontinuous harmonic maps into spheres, Acta Math. 175 (1995), 197-226.

[13] C. Wang, Biharmonic maps from $\mathbb{R}^{4}$ into a Riemannian manifold, Math. Z. 247 (2004), 65-87.

Department of Mathematical Sciences

UNIVERSITY OF BATH

BATH BA2 7AY

UK

E-mail address: r.moser@bath.ac.uk

ReCEIVEd April 9, 2013 